\title{
ARTICLE \\ Development of a prediction system for anti-tuberculosis drug-induced liver injury in Japanese patients
}

\author{
Taisei Mushiroda ${ }^{1}$, Hideki Yanai ${ }^{2,3}$, Takashi Yoshiyama ${ }^{2,3}$, Yuka Sasaki $^{2}$, Masao Okumura ${ }^{2}$, Hideo Ogata ${ }^{2,3}$ and Katsushi Tokunaga ${ }^{4}$
}

Drug-induced liver injury (DILI) is a common adverse drug reaction in patients receiving antituberculosis (anti-TB) treatment. Among the anti-TB agents, isoniazid (INH) is the primary drug that causes hepatotoxicity in TB patients with DILI. Previous reports in several populations have consistently demonstrated an association between polymorphisms in the $\mathrm{N}$-acetyltransferase 2 (NAT2) gene, which is responsible for INH hepatic metabolism, and a risk of DILI in TB patients. In this study, the genetic and baseline clinical data from 366 Japanese patients with TB (73 patients with DILI and 293 without DILI) were used to develop a system to predict DILI risk due to anti-TB agents. The distribution of the NAT2 acetylator status among the TB patients with DILI was 31 (42.5\%), 29 (39.7\%), and 13 (17.8\%) for rapid, intermediate, and slow acetylators, respectively. A significant association was observed between NAT2 slow acetylators and DILI risk (odds ratio 4.32, 95\% confidence interval $1.93-9.66, P$ value $=5.56 \times 10^{-4}$ ). A logistic regression model based on age and NAT2 genotype revealed that the area under the curve for the receiver-operating characteristic curve was 0.717 . The findings demonstrated that slow NAT2 acetylator status is a significant predictor of the risk of DILI by anti-TB agents, and a personalized anti-TB treatment approach may aid in making treatment decisions and reducing the incidence of DILI.

Human Genome Variation (2016) 3, 16014; doi:10.1038/hgv.2016.14; published online 23 June 2016

\section{INTRODUCTION}

Tuberculosis (TB) is the most prevalent chronic respiratory infection. In 2013, an estimated 9 million people developed TB worldwide, and 1.5 million patients died from the disease. ${ }^{1}$ In Japan in 2013, the incidence of TB per 100,000 people was 18 (http://www.who.int/tb/country/data/profiles/en/). A combination of four anti-TB agents-isoniazid (INH), rifampicin, pyrazinamide, and ethambutol-has been recommended as the best treatment regimen for patients with TB. ${ }^{1}$ However, drug-induced liver injury (DILI) is one of the most common adverse drug reactions in patients receiving anti-TB treatment. Previous studies have reported that DILI occurred in $6.9 \%$ of Japanese patients with TB. ${ }^{2}$ Most cases of DILI are reversible after anti-TB treatment is stopped. However, the treatment must be restarted, leading to a high prevalence of reoccurrence of DILI and a longer course of anti-TB therapy (>6 months). Eventually, hyposensitization therapy fails in a significant percentage of patients with TB. Therefore, the frequent occurrence of DILI may affect the continuation of anti-TB agent administration that leads to delayed completion or failure of the treatment.

INH is primarily metabolized to $\mathrm{N}$-acetyl INH by hepatic $\mathrm{N}$ acetyltransferase 2 (NAT2). ${ }^{3}$ Hydrazine, which is generated from $\mathrm{INH}$, is believed to be the cause of hepatotoxicity. Because NAT2 also metabolizes hydrazine to $\mathrm{N}$-acetyl hydrazine, TB patients with decreased NAT2 activity show higher levels of hydrazine in their blood and are at a higher risk of developing DILI. ${ }^{3}$ These adverse effects on the liver in TB patients are usually accompanied by hospitalization and adjustment of the anti-TB regimen that is usually less effective and requires a longer treatment duration than the standard short treatment course for TB.
Underlying differences in NAT2 enzyme activity have been explained by haplotypes composed of several single-nucleotide polymorphisms (SNPS) in the coding region of exon 2 of the NAT2 gene. ${ }^{4}$ Patients are classified into rapid, intermediate, and slow acetylators based on the codominant expression of the rapid and slow acetylator alleles. ${ }^{3}$ NAT2 rapid acetylators are homozygotes for $N A T 2 * 4$. Intermediate acetylators carry the $N A T 2^{*} 4 /{ }^{*} 5 B,{ }^{*} 4 /{ }^{*} 6 A$, or ${ }^{*} 4 /{ }^{*} 7 B$ diplotypes, whereas slow acetylators are individuals who do not carry either of the rapid acetylator alleles (NAT2*4 or NAT2*13).

Although several recent case-control association studies in Japanese TB patients have reported that NAT2 slow acetylators have a significantly higher risk of DILI caused by anti-TB drugs compared with NAT2 rapid and intermediate acetylators, the truepositive rates were $<33 \%$ when the NAT2 genotypes were used as biomarkers to predict the risk of DILI. ${ }^{5,6}$ In this study, the genetic and clinical data from 366 Japanese patients with TB (73 patients with and 293 without DILI) were used to develop a system to predict the risk of DILI by anti-TB agents.

\section{MATERIALS AND METHODS}

Patients

This study was approved by the institutional review boards of Fukujuji Hospital, RIKEN Yokohama Institute, and the University of Tokyo. All patients who participated in this study provided written informed consent.

Japanese TB patients who received anti-TB agents according to the World Health Organization category I treatment regimen (2HRZE/4HR) were enrolled in this study from Fukujuji Hospital (73 patients diagnosed with DILI and 293 tolerant controls). DILI was defined according to the World Health Organization toxicity classification standards, ${ }^{7}$ that is, elevation of aspartate aminotransferase and/or alanine aminotransferase

\footnotetext{
${ }^{1}$ Laboratory for Pharmacogenomics, RIKEN Center for Integrative Medical Sciences, Yokohama, Japan; ${ }^{2}$ Fukujuji Hospital, Japan Anti-tuberculosis Association (JATA), Kiyose, Japan; ${ }^{3}$ Research Institute of Tuberculosis, JATA, Kiyose, Japan and ${ }^{4}$ Department of Human Genetics, Graduate School of Medicine, University of Tokyo, Tokyo, Japan. Correspondence: $\mathrm{H}$ Yanai (yanaih@fukujuji.org) 
and/or total bilirubin levels more than 3 times the upper normal limit with signs and symptoms of hepatitis.

\section{SNP genotyping}

Genomic DNA was extracted from peripheral blood by a commercial laboratory (SRL, Tokyo, Japan). We sequenced four SNPs of the NAT2 gene that cover the NAT2 haplotypes commonly found in the Japanese population: rs1041983 (282C > T), rs1799929 (481C > T), rs1799930 (590G > A), and rs1799931 (857G >A). ${ }^{4}$ PCR amplification was carried out in a 20- $\mu$ l reaction mixture containing $10 \mathrm{ng}$ of genomic DNA, $0.5 \mathrm{U}$ of Tks Gflex DNA polymerase (Takara Bio, Otsu, Japan), Gflex PCR Buffer (containing $1 \mathrm{mmol} / / \mathrm{l}$ of $\mathrm{Mg}^{2+}$ and $200 \mu \mathrm{mol} / \mathrm{l}$ of $\mathrm{dNTP}$ ), and $4 \mathrm{pmol}$ of each forward and reverse primer using a GeneAmp PCR System 9700 (Applied Biosystems, Foster City, CA, USA). The following PCR conditions were used: initial denaturation at $94^{\circ} \mathrm{C}$ for $2 \mathrm{~min}, 40$ cycles at $98^{\circ} \mathrm{C}$ for $10 \mathrm{~s}, 60^{\circ} \mathrm{C}$ for $15 \mathrm{~s}, 68^{\circ} \mathrm{C}$ for $2 \mathrm{~min}$, and a final extension at $68{ }^{\circ} \mathrm{C}$ for $5 \mathrm{~min}$. Direct sequencing of the PCR products was performed on an ABI 3730xI DNA Analyzer (Applied Biosystems) using the BigDye Terminator v.3.1 Cycle Sequencing Kit according to the manufacturer's protocol.

\section{Statistical analysis}

The haplotype analysis was performed using SNPAlyze software, version 8.0.1 (Dynacom, Chiba, Japan). Association studies were conducted using Fisher's exact test. Nonparametric tests ( $X^{2}$ test and Mann-Whitney $U$-test) were used to detect significant associations between patients' characteristics and the risk of DILI caused by anti-TB agents. To identify independent factors associated with DILI, we performed a logistic regression analysis with a stepwise selection of variables. All analyses were performed using IBM SPSS Statistics software, version 22 (IBM, Armonk, NY, USA). A significance level was set at 0.05 .

\section{RESULTS}

The patient profiles are shown in Table 1. In total, 73 TB patients were diagnosed with DILI and 293 were tolerant controls. We identified four NAT2 haplotypes from the four SNPs in the Japanese TB patients (Table 2 and Supplementary Table S1). $N A T 2 * 4$ is a wild-type allele with a frequency of $70.6 \%$ in the present study; the other NAT2 alleles show decreased metabolic activity. ${ }^{3}$ Among the three slow acetylator alleles, NAT2*6A had the highest frequency (18.4\%), followed by $N A T 2^{*} 7 B(8.7 \%)$ and $N A T 2 * 5 B(2.2 \%)$. The distribution of the NAT2 alleles was significantly different between the DILI and tolerant control groups (Table 2). NAT2*6A was significantly associated with a risk of DILI (odds ratio 1.77, 95\% confidence interval 1.15-2.72, $P$ value $=1.19 \times 10^{-2}$ ).

Diplotype estimation revealed 10 diplotypes composed of the four alleles (Table 3 ), thereby demonstrating that the frequency of the variant diplotype, $N A T 2^{*} 6 \mathrm{~A} /{ }^{*} 6 \mathrm{~A}$, was significantly higher in the DILI group $(8.2 \%)$ than in the controls $(1.4 \%$, odds ratio $6.47,95 \%$ confidence interval 1.78-23.6, $P$ value $\left.=5.58 \times 10^{-3}\right)$. In addition, a higher frequency of $N A T 2^{*} 6 A{ }^{*} 7 B$ was observed in the DILI group $(5.5 \%)$ as compared with the tolerant controls $(1.7 \%)$, but this finding was not significant (Table 3 ). When the NAT2 diplotypes were classified into NAT2 rapid, intermediate, and slow acetylators, the DILI group showed a significantly higher frequency of slow acetylators (17.8\%) compared with the tolerant controls (4.8\%, odds ratio $4.32,95 \%$ confidence interval 1.93-9.66, $P$ value $=5.56 \times 10^{-4}$ ), whereas there were no significant differences in the frequency of intermediate acetylators between the DILI and the control groups (Table 4).

Table 1. Characteristics of Japanese TB patients with or without DILI caused by anti-TB agents

\begin{tabular}{|c|c|c|c|}
\hline & DILI $(\mathrm{n}=73)$ & Tolerant control $(n=293)$ & $\mathrm{P}$ value \\
\hline Sex, female/male (\%) & $16(21.9) / 57(78.1)$ & $88(30.0) / 205(70.0)$ & 0.169 \\
\hline Age, years & $64(19-87)$ & $52(18-94)$ & $5.96 \times 10^{-6}$ \\
\hline \multicolumn{4}{|l|}{ Baseline values } \\
\hline AST, U/I & $26(11-125)$ & $20(2-162)$ & $2.90 \times 10^{-4}$ \\
\hline ALT, U/I & $20(4-124)$ & $15(3-127)$ & $4.11 \times 10^{-2}$ \\
\hline Total bilirubin, mg/dl & $0.5(0.2-1.8)$ & $0.5(0.1-2.1)$ & 0.777 \\
\hline$A L P, U / I$ & $278(153-1,633)$ & $257(38-2,683)$ & 0.351 \\
\hline eGFR, $\mathrm{ml} / \mathrm{min}$ per $1.73 \mathrm{~m}^{2}$ & $83(26-153)$ & $87(17-185)$ & 0.493 \\
\hline \multicolumn{4}{|l|}{ During TB treatment } \\
\hline AST, U/I & $162(44-1,659)$ & $27(9-414)$ & $1.69 \times 10^{-34}$ \\
\hline$A L T, U / I$ & $137(10-1,555)$ & $20(3-239)$ & $1.10 \times 10^{-28}$ \\
\hline Total bilirubin, mg/dl & $0.6(0.3-5.1)$ & $0.4(0.2-15.5)$ & $1.17 \times 10^{-8}$ \\
\hline ALP, U/I & $312(159-1,097)$ & $251(87-12,328)$ & $4.31 \times 10^{-4}$ \\
\hline
\end{tabular}

Baseline values were examined before the initiation of treatment with anti-TB drugs. Counts are listed for categorical values and median and range are reported for continuous variables.

Abbreviations: ALP, alkaline phosphatase; ALT, alanine aminotransferase; AST, aspartate aminotransferase; DILI, drug-induced liver injury; eGFR, estimated glomerular filtration rate; TB, tuberculosis.

Table 2. Distribution of NAT2 haplotypes in Japanese TB patients with or without DILI

\begin{tabular}{|c|c|c|c|c|c|c|c|c|}
\hline \multirow[t]{2}{*}{ Haplotype } & \multicolumn{4}{|c|}{ SNPS } & \multicolumn{2}{|r|}{ Frequency } & \multirow[t]{2}{*}{ OR $(95 \% \mathrm{Cl})$} & \multirow[t]{2}{*}{$\mathrm{P}$ value } \\
\hline & rs1041983 & rs1799929 & rs1799930 & rs1799931 & DILI $(2 n=146)$ & Tolerant control $(2 n=586)$ & & \\
\hline$N A T 2 * 4$ & C & $C$ & G & G & 0.623 & 0.727 & $0.62(0.42-0.91)$ & $1.92 \times 10^{-2}$ \\
\hline NAT2*5B & C & $\mathrm{T}$ & G & G & 0.021 & 0.022 & $0.92(0.26-3.29)$ & 1.00 \\
\hline$N A T 2 * 6 A$ & $\mathrm{~T}$ & C & A & G & 0.260 & 0.166 & $1.77(1.15-2.72)$ & $1.19 \times 10^{-2}$ \\
\hline$N A T 2 * 7 B$ & $\mathrm{~T}$ & C & G & $A$ & 0.096 & 0.085 & $1.14(0.61-2.12)$ & 0.743 \\
\hline
\end{tabular}

Abbreviations: $\mathrm{Cl}$, confidence interval; DILI, drug-induced liver injury; NAT2, N-acetyltransferase 2; OR, odds ratio; SNP, single-nucleotide polymorphism; $\mathrm{TB}$, tuberculosis. 
The following baseline clinical factors were significantly associated with DILI: age, aspartate aminotransferase, and alanine aminotransferase. When we included all the factors significantly associated with DILI in the logistic regression analysis, age and NAT2 slow acetylator status were identified as independent predictors of the risk of DILI due to anti-TB agents (Table 5). Furthermore, the area under the curve for the receiver-operating characteristic curve was 0.717 , with a true-positive rate of $93.2 \%$ and a false-positive rate of $58.4 \%$ (Supplementary Figure S1). After the prevalence of DILI due to anti-TB agents in Japanese patients (6.9\%) was incorporated, the positive predictive value and negative predictive value of the model were 0.11 and 0.99 , respectively. ${ }^{2}$ The population attributable risk was calculated by subtracting the incidence of DILI due to anti-TB agents in the nonrisk patients from the incidence in all the patients (risk and nonrisk). The population attributable risk percent, calculated by dividing the population attributable risk by the incidence of DILI due to anti-TB agents in all the patients, was $82.5 \%$. Therefore, the probability of developing DILI can be calculated using the following prediction formula:

$$
\begin{aligned}
\log \text { odds }(\mathrm{DILI})= & -3.900+0.040 \times \text { age } \\
& +1.940 \times \text { NAT2 slow acetylator }
\end{aligned}
$$

\section{DISCUSSION}

In the present study, we observed four NAT2 haplotypes in a study population of 366 Japanese TB patients. Three of the four$N A T 2 * 5 B, N A T 2 * 6 A$, and $N A T 2 * 7 B$ - are classified as slow acetylator alleles. A significant association was observed between the NAT2 slow acetylators (individuals without NAT2*4) and DILI risk (Table 4). These findings confirm that the slow acetylator status of NAT2 is a significant risk factor for DILI caused by anti-TB agents.

Table 3. Distribution of NAT2 diplotypes in Japanese TB patients with

\begin{tabular}{|c|c|c|c|c|}
\hline Diplotype & DILI, n (\%) & $\begin{array}{l}\text { Tolerant } \\
\text { control, } \\
\text { n (\%) }\end{array}$ & OR $(95 \% \mathrm{Cl})$ & $\mathrm{P}$ value \\
\hline NAT2*4/*4 & $31(42.5)$ & $147(50.2)$ & $0.73(0.44-1.23)$ & 0.295 \\
\hline$N A T 2 * 4 / * 5 B$ & $1(1.4)$ & $8(2.7)$ & $0.49(0.06-4.02)$ & 0.694 \\
\hline$N A T 2 * 4 /{ }^{*} 6 A$ & $21(28.8)$ & $83(28.3)$ & $1.02(0.58-1.80)$ & 1.00 \\
\hline$N A T 2 * 4 /{ }^{*} 7 B$ & $7(9.6)$ & $41(14.0)$ & $0.65(0.28-1.52)$ & 0.438 \\
\hline$N A T 2 * 5 B / * 5 B$ & $0(0.0)$ & $2(0.7)$ & NA & 1.00 \\
\hline NAT2 ${ }^{*} 5 B /{ }^{*} 6 A$ & $1(1.4)$ & $1(0.3)$ & $4.06(0.25-65.6)$ & 0.360 \\
\hline$N A T 2 * 5 B / * 7 B$ & $1(1.4)$ & $0(0.0)$ & NA & 0.199 \\
\hline$N A T 2 * 6 A{ }^{*} 6 A$ & $6(8.2)$ & $4(1.4)$ & $6.47(1.78-23.6)$ & $5.58 \times 10^{-3}$ \\
\hline$N A T 2 * 6 A{ }^{*} 7 B$ & $4(5.5)$ & $5(1.7)$ & $3.34(0.87-12.8)$ & 0.0824 \\
\hline$N A T 2^{*} 7 B /{ }^{*} 7 B$ & $1(1.4)$ & $2(0.7)$ & $2.02(0.18-22.6)$ & 0.488 \\
\hline
\end{tabular}
or without DILI

Abbreviations: $\mathrm{Cl}$, confidence interval; DILI, drug-induced liver injury; NA, not available; NAT2, N-acetyltransferase 2; OR, odds ratio; TB, tuberculosis.
The frequency of $N A T 2 * 6 A / 6 A$ was significantly higher in the DILI group (8.2\%) compared with the tolerant controls $(1.4 \%)$, whereas there was a higher frequency of the $N A T 2^{*} 6 A{ }^{*} 7 B$ haplotype in the DILI group (5.5\%) compared with the controls (1.7\%), although the difference was not significant (Table 3$)$. In previous reports, subjects with the $N A T 2^{*} 6 A /{ }^{*} 6 A$ and $N A T 2^{*} 6 A /{ }^{*} 7 B$ haplotype demonstrated lower $N$-acetyltransferase activity compared with the other slow acetylator haplotypes. ${ }^{8,9}$ Selinski et al. ${ }^{9}$ investigated the risk of bladder cancer associated with NAT2 dyplotypes and found that $N A T 2^{*} 6 A{ }^{*} 6 A, N A T 2^{*} 6 A /^{*} 7 B$, and $N A T 2^{*} 7 B /{ }^{*} 7 B$ should be classified as "ultra-slow" acetylators, according to their in vivo caffeine $N$-acetyltransferase activity compared with other slow acetylators. In the present study, TB patients with the $N A T 2^{*} 6 A{ }^{*} 6 A$ haplotype showed a higher odds ratio and a lower $P$ value compared with patients with the $N A T 2 * 6 A{ }^{*} 7 B$ haplotype, indicating that $N A T 2 * 6 A$ might be superior as a biomarker to other NAT2 alleles; however, this finding is not conclusive because of the small sample size. Future studies using meta-analysis will be conducted to compare the NAT2 "ultraslow" acetylators in other ethnic groups. In addition, we will conduct a pharmacokinetic study to examine the correlation between the NAT2 diplotypes and the levels of INH and hydrazine in the plasma of patients with TB.

Although NAT2 slow acetylator status would be expected to be the best predictor for the risk of DILI, true-positive rates were reported to be $<33 \%$ when the NAT2 genotypes were used as biomarkers to predict DILI in Japanese TB patients. ${ }^{5,6}$ The inclusion of baseline clinical data should improve the true-positive rate for the prediction of DILI. In this study, we demonstrated that age was a clinical predictive factor for DILI due to anti-TB agents and developed a simple predictive model incorporating age and genetic variables. The model improved the true-positive rate from $17.8 \%$ (when applied only NAT2 slow acetylator status) to $93.2 \%$. In addition, the population attributable risk percent values of the models using the two variables and of the model using only the NAT2 slow acetylator status were $82.5 \%$ and $12.9 \%$, respectively. This indicates that there is a higher potential impact of the model using the clinical and genetic variables compared with the model using only the genetic variable.

The calculated positive predictive value (0.11) indicated that the prediction system could identify 11 patients at high risk of DILI by anti-TB agents per 100 Japanese TB patients, and the estimated number needed to test was 9 . Whether this number needed to test is high or low depends on many factors, such as the cost of

Table 5. Multivariate predictors for risk of DILI due to anti-TB agents

\begin{tabular}{lccc}
\hline Variable & Coeff & OR $(95 \% \mathrm{Cl})$ & P value \\
\hline Age & 0.040 & $1.04(1.02-1.06)$ & $1.53 \times 10^{-6}$ \\
NAT2 slow acetylator & 1.940 & $6.96(2.86-17.0)$ & $1.93 \times 10^{-5}$ \\
\hline
\end{tabular}

Abbreviations: $\mathrm{Cl}$, confidence interval; Coeff, coefficient; DILI, drug-induced liver injury; OR, odds ratio; NAT2, N-acetyltransferase 2; TB, tuberculosis.

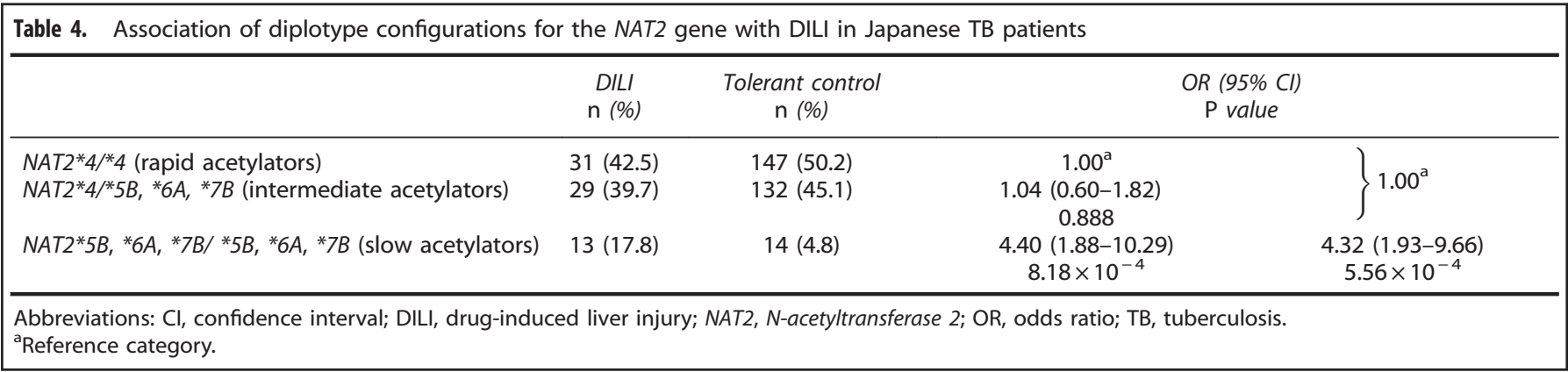


genetic testing. Therefore, in the future, an economic evaluation should be conducted from the perspective of a national health insurance system in Japan. In addition, the cost of genetic testing should be taken into consideration to determine whether both NAT2 status and age information are necessary for the prediction formula; however, a higher true-positive rate is more desirable from the viewpoint of patient safety.

For patients with the INH-resistant strain of Mycobacterium tuberculosis, levofloxacin has long been recommended by the Japanese Society of Tuberculosis and other academic societies. Recently, levofloxacin was approved as an anti-TB agent in Japan. Other new drugs, such as delamanid, have already been approved in Japan. Another new drug, betaquiline, has been approved in many countries, and an application has been filed for approval in Japan. Therefore, these drugs may be used as an alternative to INH for patients at high risk of liver injury. In addition to the use of alternative drugs, the results of the DILI risk predictions suggest that dosing stratification of INH may be beneficial for TB patients.

In conclusion, we confirmed that the NAT2 alleles significantly contribute to susceptibility to DILI due to anti-TB agents in a Japanese population. We also developed a predictive model incorporating genetic and clinical risk factors to assess DILI risk. Future prospective clinical trials are necessary to ascertain the clinical utility of this model. These trials will provide clinical evidence for INH dosage adjustment and lead to a reduction in the incidence of DILI and improvement in the efficacy of anti-TB treatment.

\section{ACKNOWLEDGEMENTS}

We thank all the patients who enrolled in our study. This work was supported by grants-in-aid from the Ministry of Education, Culture, Sports, Science, and Technology of the Japanese Government (JSPS KAKENHI grant number 24406010, 15H05271, and BioBank Japan Project). This research was also supported by AMED/JICA, SATREPS. We express our gratitude to Aiko Ohno and Mitsunori Yahata for their excellent technical support.

\section{AUTHOR CONTRIBUTIONS}

Conceived and designed the experiments: TM, HY, and KT. Collection of samples: HY, TY, YS, MO, and HO. Performed experiments: TM and HY. Data analysis: TM and HY. Drafting the paper: TM, HY, and KT.

\section{COMPETING INTERESTS}

The authors declare no conflict of interest.

\section{REFERENCES}

1 World Health Organization. Global tuberculosis report 2014. Geneva, Switzerland.

2 Kaneko Y, Nagayama N, Kawabe Y, Shimada M, Suzuki J, Kunogi M et al. Drug-induced hepatotoxicity caused by anti-tuberculosis drugs in tuberculosis patients complicated with chronic hepatitis. Kekkaku 2008; 83: 13-19.

3 McDonagh EM, Boukouvala S, Aklillu E, Hein DW, Altman RB, Klein TE. PharmGKB summary: very important pharmacogene information for $\mathrm{N}$-acetyltransferase 2 . Pharmacogenet Genomics 2014; 24: 409-425.

4 Taniguchi A, Urano W, Tanaka E, Furihata S, Kamitsuji S, Inoue E et al. Validation of the associations between single nucleotide polymorphisms or haplotypes and responses to disease-modifying antirheumatic drugs in patients with rheumatoid arthritis: a proposal for prospective pharmacogenomic study in clinical practice. Pharmacogenet Genomics 2007; 17: 383-390.

5 Ohno M, Yamaguchi I, Yamamoto I, Fukuda T, Yokota S, Maekura R et al. Slow $\mathrm{N}$-acetyltransferase 2 genotype affects the incidence of isoniazid and rifampicininduced hepatotoxicity. Int J Tuberc Lung Dis 2000; 4: 256-261.

6 Higuchi N, Tahara N, Yanagihara K, Fukushima K, Suyama N, Inoue Y et al. NAT2*6A, a haplotype of the $\mathrm{N}$-acetyltransferase 2 gene, is an important biomarker for risk of anti-tuberculosis drug-induced hepatotoxicity in Japanese patients with tuberculosis. World J Gastroenterol 2007; 13: 6003-6008.

7 Tostmann A, Boeree MJ, Aarnoutse RE, de Lange WC, van der Ven AJ, Dekhuijzen R. Antituberculosis drug-induced hepatotoxicity: concise up-to-date review. J Gastroenterol Hepatol 2008; 23: 192-202.

8 Cascorbi I, Drakoulis N, Brockmöller J, Maurer A, Sperling K, Roots I. Arylamine $\mathrm{N}$-acetyltransferase (NAT2) mutations and their allelic linkage in unrelated Caucasian individuals: correlation with phenotypic activity. Am J Hum Genet 1995; 57: 581-592.

9 Selinski S, Blaszkewicz M, Ickstadt K, Hengstler JG, Golka K. Refinement of the prediction of $\mathrm{N}$-acetyltransferase 2 (NAT2) phenotypes with respect to enzyme activity and urinary bladder cancer risk. Arch Toxicol 2013; 87: 2129-2139.

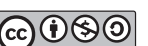

This work is licensed under a Creative Commons AttributionNonCommercial-ShareAlike 4.0 International License. The images or other third party material in this article are included in the article's Creative Commons license, unless indicated otherwise in the credit line; if the material is not included under the Creative Commons license, users will need to obtain permission from the license holder to reproduce the material. To view a copy of this license, visit http:// creativecommons.org/licenses/by-nc-sa/4.0/

(c) The Author(s) 2016

Supplementary Information for this article can be found on the Human Genome Variation website (http://www.nature.com/hgv). 